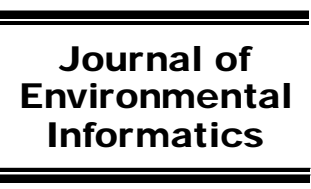

www.iseis.org/jei

\title{
Modeling Chlorobenzene Leaching from a Landfill into a Soil Environment Using Particle Filter Approach
}

\author{
S. Y. Chang* and X. P. Li \\ Civil and Environmental Engineering Department, North Carolina A\&T State University, Greensboro, North Carolina 27411, USA
}

Received 25 January 2008; revised 3 March 2007; accepted 13 March 2008; published online 1 December 2008

\begin{abstract}
With increased use and public awareness of landfills, there is much concern for the pollution potential of the landfill leachate. As leachate migrates away from a landfill, it may cause serious pollution to the groundwater aquifer as well as adjacent surface waters. Modeling the behavior of pollutants during the flow of leachate through soil is essential for predicting the fate of these pollutants and designing restoration strategies. In this paper, Particle filter approach is introduced for the estimation of chlorobenzene leachating from a landfill into a soil environment. The discrete advection transport equation is used as a deterministic model, in which the state vector denotes the concentrations at discrete nodes. First, random Gaussian errors are added to the transport equation to become the state equation. Then, the Particle filter recursive process is applied for that state space model. In each time step, random samples which represent the density of state vector are propagated through state equations and updated by observation data assimilation. The result shows that the filtered estimation from Particle filter approach is in close agreement with the true value. Furthermore, the efficiency of Particle filter approach is compared with the numerical method using root mean square error (RMSE). The RMSE value for the numerical method was seven times larger than that for the Particle filter approach.
\end{abstract}

Keywords: landfill leachate; particle filter; state space model; subsurface transport model; state estimation

\section{Introduction}

Sequential state estimation is the process of using dynamic data from a system to estimate quantities that give a complete description of the state of the system. State estimation has the potential to be widely applied in water quality modeling. For instance, it can be used in subsurface contaminant transport problems, in dissolved oxygen (DO) estimation in ecological system such as a river or a lake and, more generally, it can be used for water quality data assimilation. For subsurface contaminant transport, state estimation can provide crucial information to describe the contamination within subsurface circumstance whose properties vary with time and space. For the river or lake system, state estimation may help to understand how the water quality is influenced by ecological processes such as photosynthesis and respiration of micro algal biomass.

State estimation can be considered as an optimal filtering problem within a Bayesian framework. State-estimation methodologies, based on Bayesian framework are powerful because (a) they are rigorously based on the probability axioms and therefore preserve information, and (b) they give the probability density function (PDF) of the model state conditioned

\footnotetext{
* Corresponding author. Tel.: +1 336 3347737; fax: +1 3363347126.

E-mail address: Chang@ncat.edu (S. Y. Chang).
}

ISSN: 1726-2135 print/1684-8799 online

(c) 2008 ISEIS All rights reserved. doi:10.3808/jei.200800127 on the available information, which may then be used for any probability-based water quality problems. With the PDF available, we can not only estimate the state but also give a description of the associated uncertainties. It is more reasonable to make prediction with uncertainty since a model, its initial and boundary conditions, and field measurements contain errors. The most well known Bayesian state-estimation algorithm is the Kalman filter (KF) (Kalman et al., 1961), which is applicable for linear models with Gaussian uncertainties. Later, KF was modified to extended Kalman filter to accommodate nonlinear system. For the last 30 years, the Kalman filter and extended Kalman filter have been applied to meteorological data assimilation (Ghil et al., 1981; Gustafsson, 1981; Houtekamer and Michell, 1998), to surface and subsurface hydrologic estimation (Bowles, 1978; Van Geer, 1982; Yu et al., 1989; Yangxiao et al., 1991; Ferraresi and Marinelli, 1996; Harrouni et al., 1997; Porter et al., 2000; McLaughlin, 2002), to subsurface contaminant transport estimation (Cheng, 2002; Chang and Jin, 2005), and to water quality modeling in river circumstance (Beck and Young, 1976; Cosby et al., 1984; Whitehead and Hornberger, 1984).

During last decade, the Particle filter, based on sequential Monte Carlo method, has been introduced to state estimation in nonlinear systems and successfully applied in a number of different areas such as electronic engineering, ecosystem modeling, structure modeling, and chemical process modeling (Gordon et al., 1993; Kitagawa, 1996; Doucet et al., 2000; Svetlana et al., 2003; Chen et al., 2005; Ching et al., 2005). 
Particle filter application for state and parameter estimation in nonlinear structure model was studied by Ching et al. (2005). Chen et al. (2005) applied the Particle filter to the modeling of a benchmark batch polymerization process. The on-line state and parameters for the process are estimated. However, there has been limited application of Particle filters in subsurface contaminant transport modeling.

The Particle filter is potentially effective for estimation and data assimilation problems in subsurface contaminant transport due to its advantages in dealing with dynamic and stochastic processes. The objective of the paper is to apply Particle filters for contaminant transport estimation in a soil environment and evaluate its performance. The rest of this paper has the following structure: In section 2, the deterministic transport model, the Bayesian state estimation method and the Particle filter algorithm will be introduced; In section 3 , discussion will focus on some practical aspects which are related to application of Particle filter in this particular study; In sections 4 and 5, the Particle filters are demonstrated by application to a state-space model for state estimation, and its performance is evaluated by the root mean square error (RMSE).

\section{Methodology}

\subsection{1-Dimensional Contaminant Transport Equation and the State-Space Model}

The transport equation for contaminant in a soil environment with the direction of flow parallel to the y-axis is:

$\frac{\partial C}{\partial t}=-\frac{V}{R} \frac{\partial C}{\partial y}$

where

$C=$ Solute concentration, $\mathrm{mg} / \mathrm{l}$;

$t=$ Time, sec;

$y=$ Cartesian coordinates, $\mathrm{m}$;

$V=$ Linear velocity of flow field in the y direction, $\mathrm{m} / \mathrm{sec}$;

$R=$ Retardation factor, dimensionless.

The retardation factor $R$ :

$R=1+\frac{\rho_{b}}{\theta} k_{d}$

where

$\rho_{b}=$ Dry bulk mass density of the soil, $\mathrm{kg} / \mathrm{m}^{3}$;

$\theta=$ Soil porosity, dimensionless;

$k_{d}=$ Linear distribution coefficient for the solute with the soil, $\mathrm{m}^{3} / \mathrm{kg}$.

In our experimental models, it is assumed that an initial spontaneous point mass source at $(t=0, y=0)$ and a boundary condition:

$\left.C(y, t)\right|_{t=0, y=0}=C_{0}$
$\left.C(y, t)\right|_{\Omega}=0$

where $C_{0}$ is the initial concentration at $(t=0, y=0) ; \Omega$ is chosen as a boundary in this study.

Before applying Particle Filter scheme to this problem, the mathematical model that simulates the dynamic process of pollutant transport needs to be represented as a state-space form. To realize that, a finite-difference scheme is used to discretizating the space into $n$ nodes and the time into $m$ time steps. Then the Forward-Time and Central-Space (FTCS) method is applied to the original transport equation:

$\frac{\partial C}{\partial t} \approx \frac{C(i, t+1)-C(i, t)}{\Delta t}$

$\frac{\partial C}{\partial y} \approx \frac{C(i+1, t)-C(i-1, t)}{2 \Delta y}$

where

$i=1,2,3, \ldots, n$;

$t=1,2,3, \ldots, m$;

$n=$ Total number of discrete nodes;

$m=$ Total number of time steps.

Substituting equations (5) and (6) into the 1-dimentsional subsurface transport equation (1) gives:

$C_{i, t+1}=\phi_{1} C_{i-1, t}+\phi_{2} C_{i, t}+\phi_{3} C_{i+1, t}$

where

$C_{i, t}=$ Vector of pollutant concentration at node $i$ and time $t$;

$C_{i, t+1}=$ Vector of pollutant concentration at node $i$ and time $t+1$;

$\phi_{1}=\Delta t V /(2 \Delta y R)$;

$\phi_{2}=1$;

$\phi_{3}=-\Delta t V /(2 \Delta y R)$.

Assuming the number of nodes $n$ is 5 , then the statespace model is:

$\left[\begin{array}{l}C_{1, t} \\ C_{2, t} \\ C_{3, t} \\ C_{4, t} \\ C_{5, t}\end{array}\right]=\left[\begin{array}{ccccc}1 & 0 & 0 & 0 & 0 \\ \frac{\Delta t V}{2 \Delta y R} & 1 & -\frac{\Delta t V}{2 \Delta y R} & 0 & 0 \\ 0 & \frac{\Delta t V}{2 \Delta y R} & 1 & -\frac{\Delta t V}{2 \Delta y R} & 0 \\ 0 & 0 & \frac{\Delta t V}{2 \Delta y R} & 1 & -\frac{\Delta t V}{2 \Delta y R} \\ 0 & 0 & 0 & \frac{\Delta t V}{2 \Delta y R} & 1\end{array}\right] \cdot\left[\begin{array}{c}C_{1, t-1} \\ C_{2, t-1} \\ C_{3, t-1} \\ C_{4, t-1} \\ C_{5, t-1}\end{array}\right]$

\subsection{Bayesian Approach to Dynamic State Estimation}

In estimation problems the task is to estimate unknown 
quantities from noisy observations, often with prior knowledge available. Therefore, it is natural to use a Bayesian approach. Many engineering problems are by nature recursive and require on-line solutions.

In order to analyze and make inference about a dynamic system, at least two models are required (Arulampalam et al., 2002). First, a model describing the evolution of the state with time (the system model) and, second, a model relating the noisy measurements to the state (the observation model).

The equations describing the states of the systems may be combined into one vector matrix equation of the form:

$x_{t}=f\left(x_{t-1}, v_{t-1}\right)$

where $x_{t}$ is a time-variable n-dimensional vector of state variables; $v_{t-1}$ is an n-dimensional vector of random process noise; $f$ is the possible function of the states $x_{t-1}$ and $v_{t-1}$.

If we take a series of measurements at discrete time steps $t, t+1, t+2, \ldots$, the measurement model is then:

$z_{t}=h\left(x_{t}, \varepsilon_{t}\right)$

where $z_{t}$ is an n-dimensional vector of states observations at time $t ; \varepsilon_{t}$ is an n-dimensional vector of random errors.

A simplified model can be developed if the functions in Equations (9) and (10) are linear:

$x_{t}=f\left(x_{t-1}\right)+v_{t-1}$

$z_{t}=h\left(x_{t}\right)+\varepsilon_{t}$

The Bayesian approach to dynamic state estimation consists of essentially two stages: prediction and update. The prediction stage uses the system model to predict the state probability density function (pdf). The update operation uses the latest measurement to modify the prediction pdf. This is achieved by using the Bayes theorem, which is the mechanism for updating knowledge about the target state in the light of extra information from new data.

The estimation $x_{t}$ from measurements, from Bayesian perspective, is to calculate the degree of belief in the state $x_{t}$ at time $t$, given the data $z_{1: t}$. Thus it is required to construct the pdf $p=p\left(x_{t} \mid z_{1: t}\right)$.

The Bayesian estimation formulation consists of the time update equation (11) and the measurement update equation (12).

$$
\begin{aligned}
& p\left(x_{t} \mid z_{1: t-1}\right)=\int p\left(x_{t} \mid x_{t-1}\right) p\left(x_{t-1} \mid z_{1: t-s}\right) d x_{t-1} \\
& p\left(x_{t} \mid z_{1: t}\right)=\frac{p\left(z_{t} \mid x_{t}\right) p\left(x_{t} \mid z_{1: t-1}\right)}{p\left(z_{t} \mid z_{1: t-1}\right)}
\end{aligned}
$$

For a simplified model that Equations (11) and (12) describe, the following relations can be calculated:

$$
\begin{aligned}
& p\left(x_{t+1} \mid x_{t}\right)=p_{v}\left(x_{t+1}-f\left(x_{t}\right)\right) \\
& p\left(z_{t} \mid x_{t}\right)=p_{\varepsilon}\left(z_{t}-h\left(x_{t}\right)\right)
\end{aligned}
$$

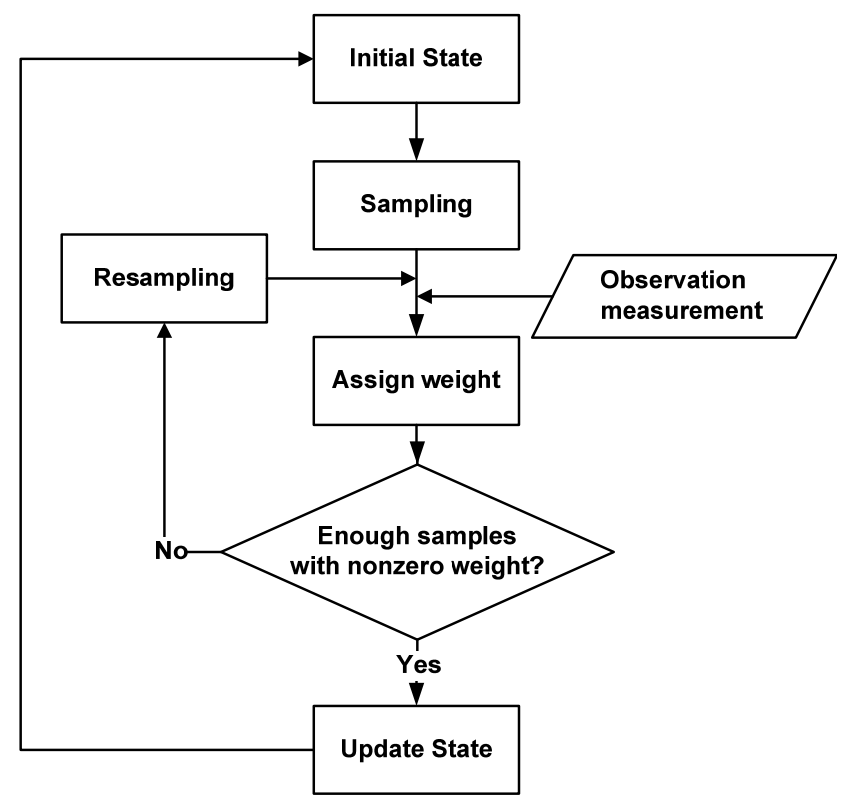

Figure 1. The recursive process of the particle filter algorithm.

\subsection{Particle Filter Algorithms}

Particle filters are an extension to point-mass filters. The basic idea of Particle filters is to approximate $p\left(x_{t} \mid z_{1: t}\right)$ using a set of random samples (also called particles) $\left\{x_{t}^{i}, i=1\right.$, $2, \ldots, N\}$ with associated weights $\left\{w_{t}^{i}, i=1,2, \ldots, N\right\}$ where $\sum_{i=1}^{N} w_{t}^{i}=1$ (Doucet et al., 2000):

$p\left(x_{t} \mid z_{1: t}\right) \approx \sum_{i=1}^{N} w_{t}^{i} \delta\left(x_{t}-x_{t}^{i}\right)$

where $\delta(x)$ is an indicator function which is equal to unity if $x=0$; otherwise it is equal to zero.

The location and weight of each particle reflect the value of the density in that region of the state space. With these particles and associated weights, the estimated state vector, $\hat{x}_{t}$, is the mean of $p\left(x_{t} \mid z_{1: t}\right)$ and is calculated as:

$\widehat{x}_{t}=\sum_{i=1}^{N} w_{k}^{i} x_{i}^{i}$

Then Particle filter updates the particle locations and the corresponding weights recursively with each new observation. However, this approach leads to divergence, if most of the 
particles have a zero weight. This divergence problem can be solved by introducing a selection or resampling step. The general Particle filter estimation recursive process is shown in Figure 1.

\subsection{Particle Filter Effectiveness Examination}

The effectiveness of the Particle filter can be shown by comparing the results from the models with and without the Particle filter. The root mean squared error (RMSE) is used to as error parameter RMSE $(k)$ :

$$
\operatorname{RMSE}(k)=\sqrt{\frac{1}{N_{i}-1} \sum\left[C^{E}(i, k)-C(i, k)\right]^{2}}
$$

where

$R M S E(k)$ : the error for time step $k$;

$C^{E}(i, k)$ : the expectation for the concentration at node $i$ at time step $k$;

$C(i, k)$ : the estimated concentration at node $i$ at time step $k$;

$N_{i}$ : the number of nodes.

Each RMSE value shows the error associated with all 10 nodes in the specific time step $k$, so RMSE is the function of time.

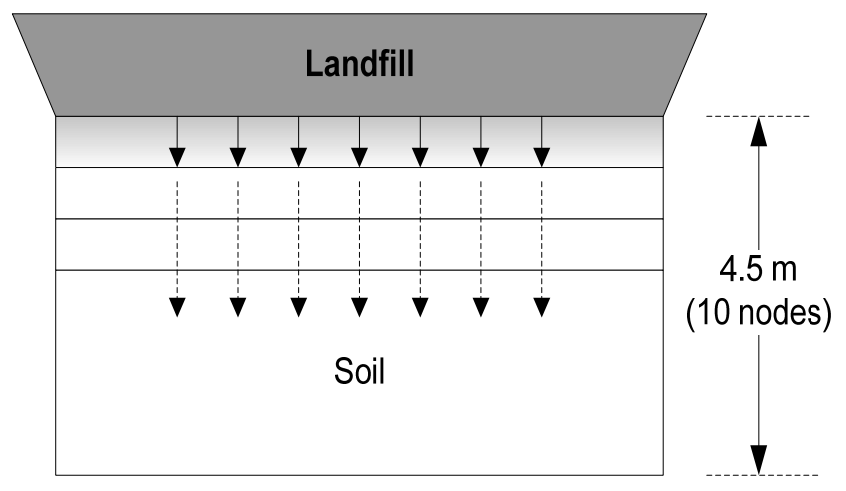

Figure 2. The application of discrete-space approach on the one-dimensional problem.

\section{Example Application}

\subsection{Application Scenario}

By applying the discrete-space approach, a one-dimensional numerical scheme is constructed for simulation the contaminant advection process in a soil environment. The model grid $G_{m}$ is defined on a 1-D plane domain (Figure 2). $G_{m}$ has $n$ grid points in $y$ direction. In the numerical scheme, we set $n$ $=10, d y=0.5 \mathrm{~m}, d t=0.20$ day. In this problem, it is assumed that: (a) each soil layer is homogenous; (b) isothermal conditions prevail; (c) soil layer is completely saturated; (d) Darcy's Law is valid; (e) no biological activity is affecting the contaminate migration.
Table 1. The Hydraulic Parameters of the Soil

\begin{tabular}{lll}
\hline Parameters & From Fernandes (1996) & In this study \\
\hline Effective porosity & $22-45 \%$ & $30 \%$ \\
Bulk dry density & $60-480 \mathrm{~kg} / \mathrm{m}^{3}$ & $300 \mathrm{~kg} / \mathrm{m}^{3}$ \\
Hydraulic conductivity & $1 \times 10^{-5}-1 \times 10^{-8} \mathrm{~m} / \mathrm{s}$ & $5 \times 10^{-7} \mathrm{~m} / \mathrm{s}$ \\
\hline
\end{tabular}

The hydraulic parameters for the soil environment are listed in Table 1 . The initial concentration $C_{0}$, which is injected into the top node, is $500 \mathrm{mg} / \mathrm{l}$ in this study.

\subsection{Practical Aspects of Implementing Particle Filter}

In order to implement the Particle filter algorithm for the given transport model and sets of parameter values in Table1, four kinds of errors and the "true value" must be specified:

\subsubsection{The "true" Data and Its Error}

Based on the same numerical model used in the predicttion, the true value is produced by adding random errors into the prediction result from the numerical model. Those random errors indicate the uncertainty in a real world. Since the random error cannot be duplicated by using computer programs, the profile of true value will vary slightly for each different run but with the basic shape. The error in true value field in this study is assumed to follow the Gaussian distribution with zero mean and standard deviation $5 \%$.

\subsubsection{The Initial Error}

The initial error covariance matrix is usually treated as a diagonal matrix. The only elements that need be specific initially are the diagonal elements which can be interpreted as the uncertainty in the initial estimates of state variable. The initial errors are chosen arbitrarily since the system error will theoretically converge to the true estimation error as long as the system equation is corrected. The concentration of $10 \mathrm{mg} / \mathrm{l}$ is used as the initial error in this study.

\subsubsection{The Error in the System Equation}

The system error covariance matrix is a diagonal matrix. The zero off-diagonal elements indicate assumed independence of the disturbances affecting each state. The elements of system error matrix corresponding to state variables indicate uncertainty in state estimates arising from several sources: random disturbances in the system, errors or random disturbances in measured inputs, and to some extent the error inherent in representing a complex system with a simple model. Value of diagonal elements is difficult to obtain. In this study, Gaussian error with $\sigma_{\text {sys }}=2.5 \%$ is chosen for indicating the uncertainty in system equation.

\subsubsection{The Error in the Observation Equation}

The observation error matrix is a diagonal matrix. The diagonal elements are the measure of the inherent uncertainty 
of the state observations. Usually they are obtained from the known precision and accuracy the instrument or technique used to the measure the state variable. Estimations of the precision are easily obtained from laboratory steadies or from the literature; $\sigma_{o b s}=2.5 \%$ is used as standard deviation in this study.

\section{Results and Discussion}

\subsection{Prediction from Numerical Model}

Firstly, the FTCS numerical method is applied to the original deterministic model with specified boundary and initial conditions as described in the section above. The result of the first step is presented in Figure 3, which shows the concentration profile predicted by the numerical method without observation correction. As shown, at the grid with time $=0$ and distance $=0$, the pollutant concentration is equal to the initial concentration $500 \mathrm{mg} / \mathrm{l}$ and there is no other girds with concentration. Along the timeline, the "peak" in concentration profile moves along from the first node to the lower nodes, which indicates the velocity direction of pollutant transport is from the top layer of soil to the lower layer. The smooth curve shows the theoretical one dimensional advection transport process.

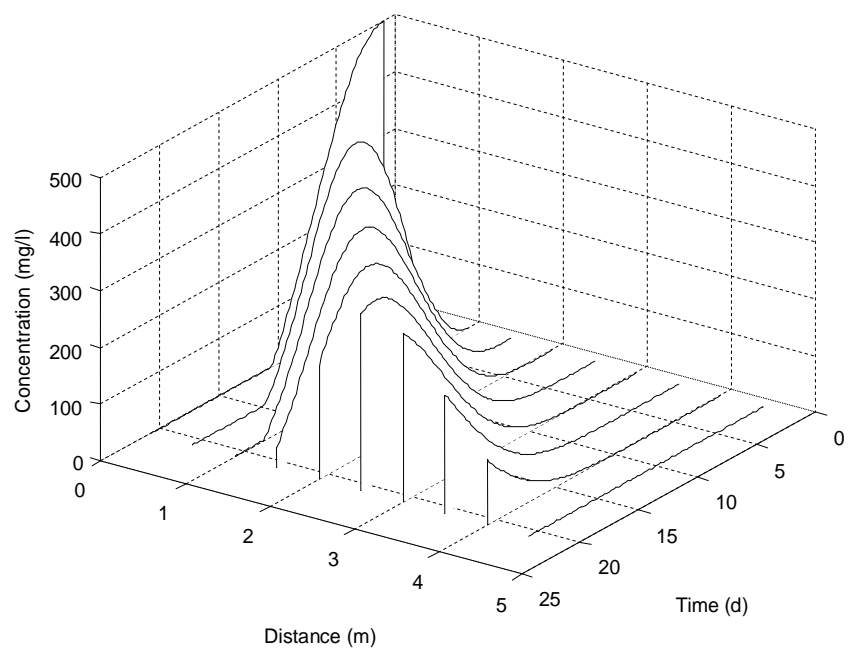

Figure 3. Predicted chlorobenzene concentration profile from the numerical method.

\subsection{Uncertainty Simulation in "True field"}

To simulate the uncertainty and inaccuracy in the true field, the random numbers drawn from the normal distributions are incorporated into the result from the numerical method. Figure 4 shows the one of 3-dimensional profile of the concentration after the random uncertainty is taken into consideration. Compared to the simulation result from the numerical method, the "true field" curve is more irregular due to the random errors. Every time the program is run, we get the different random numbers with the same distribution; the true field cannot be repeated.

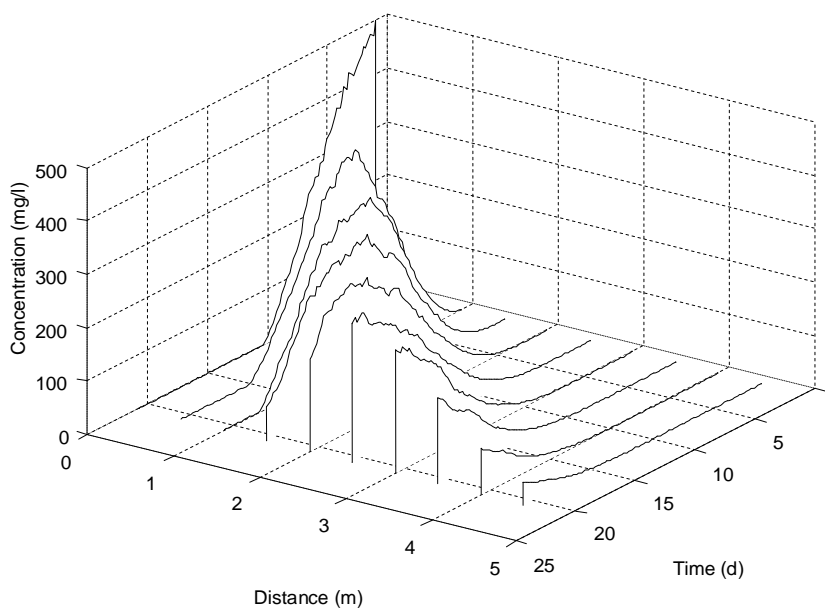

Figure 4. The true value field of chlorobenzene concentration.

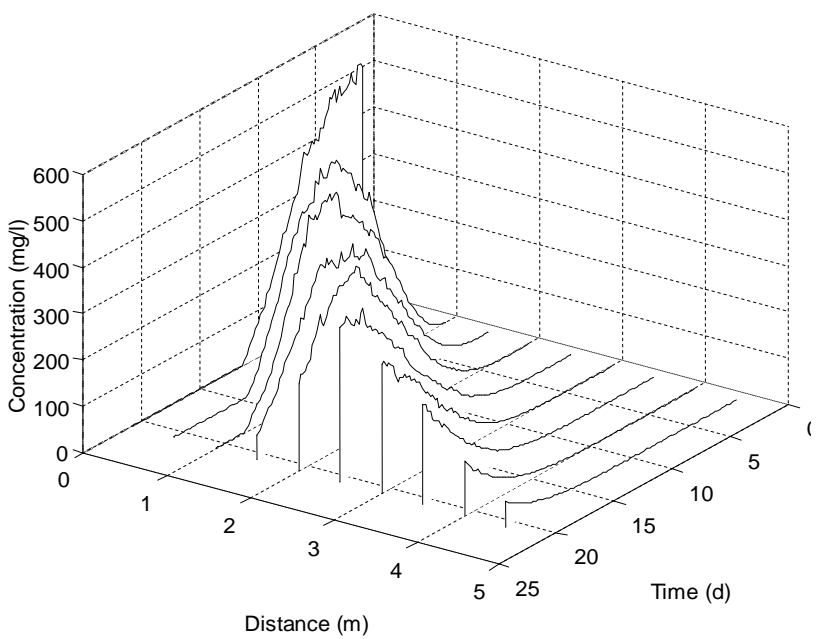

Figure 5. Predicted chlorobenzene concentration profile from Particle filter.

\subsection{Prediction from Numerical Model with Observation Correction}

As mentioned in section "practical issue for applying Particle filter”, the random errors, assumed as Gaussian distribution with zero mean and standard deviations $10 \%$, are inserted to the Equation (7). The complete system equation indicates the system evolves with time and the uncertainty in this process. Based on the system equation, the same time series is repeatedly processed, corrected by the observation data by using the Particle filter algorithm. The observation data, with assumed error, is used for this data assimilation process. In each time step, 500 random samples are drawn from the prior density function. Each sample is then assigned a weight based on the likelihood density function. Figure 5 presents the 3-dimentional predicted concentration profile by using Particle filter. By assimilating observation data from the field nodes into the state equation, the Particle filter reduces the model deviation and the resulting stochastic model makes the improved prediction. 
The RMSE for the numerical method and Particle filter method is shown in Figure 6. As shown in Figure 6, without correction of observation data, the prediction of the deterministic transport model tends to depart from the true field of stochastic process along the timeline. The calculated RMSE (for numerical method) varies with time step. And the maximum RMSE for numerical method is shown as $37 \mathrm{mg} / \mathrm{l}$ in the 11th day. The effectiveness of the Particle filter is also verified by the RMSE value in each time step. As shown in Figure 6 , the RMSE for the predicted result from Particle filter algorithm is around $5 \mathrm{mg} / \mathrm{l}$ along with the time line, which is seven times lower than the maximum error in the predicted result from the FTCS numerical method.

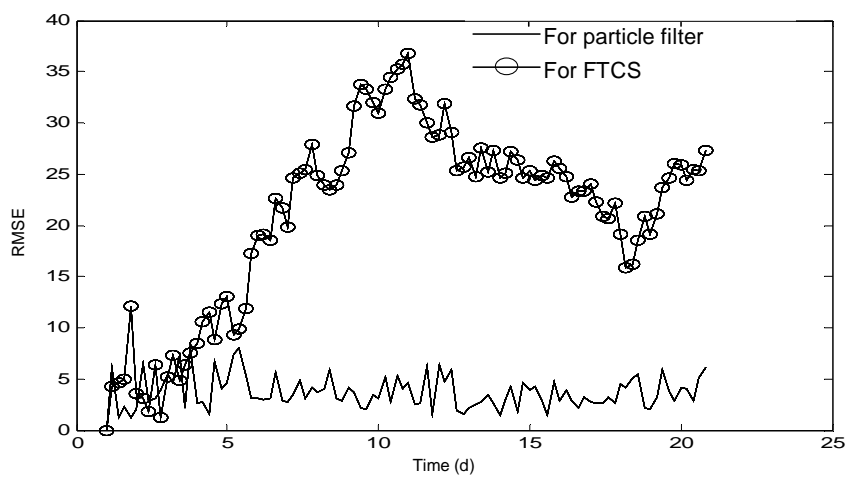

Figure 6. Prediction error for numerical method and Particle filter method.

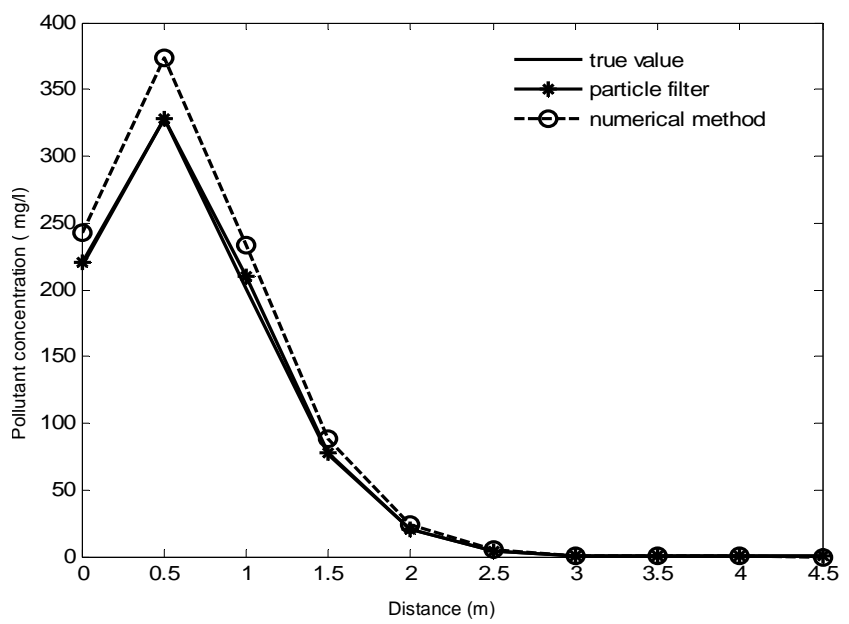

Figure 7. The concentration profile along with distance at time $=4$ day.

\subsection{The Predicted Results at Fixed Time Step}

Now we investigate the prediction process further by fixing the time step. Figure 7 shows the assimilated concentration from the numerical method and Particle filter algorithm along with the distance on the 4th day. There is no obvious difference between the results from two methods. This result may due to the less amount of observation data used for the assimilating process at the beginning of the experiment. At the time 20th day, the prediction result from Particle filter, which is corrected by observation data, seems more close to the "true value" than that from the numerical method. As Figure 8 shows, the shape of line with circle (indicating the result from Particle filter) fits the true value field well in each node. Figures 7 and 8) show that the effect of Particle filter will become significant only after the system equation is corrected by enough observation data along with the timeline. In this experiment, the "threshold" is around the 4th day. This is also verified by the Figure 6 , which presents the small difference between two methods in the first 4 days and a bigger difference after 4th day till the end of experiment.

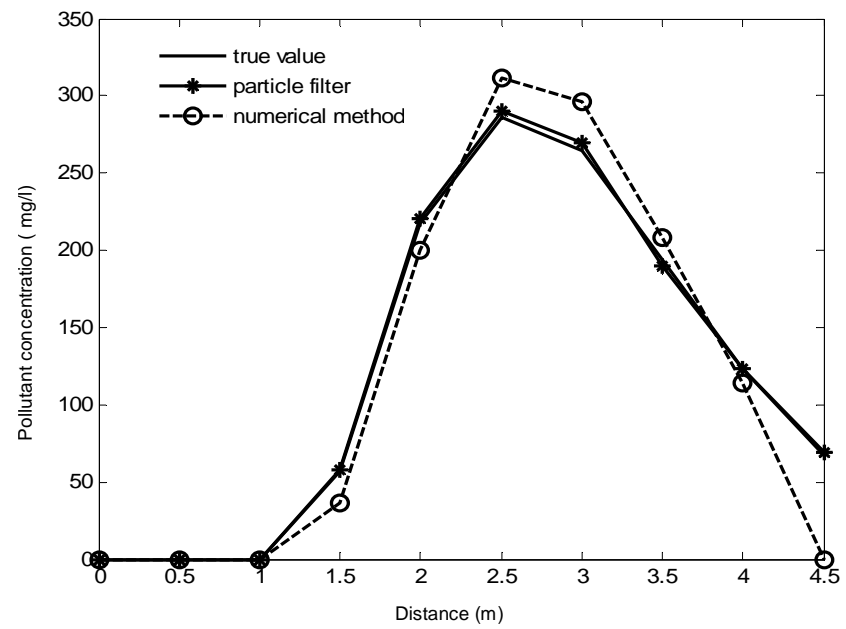

Figure 8. The concentration profile along with the distance at time $=20$ day.

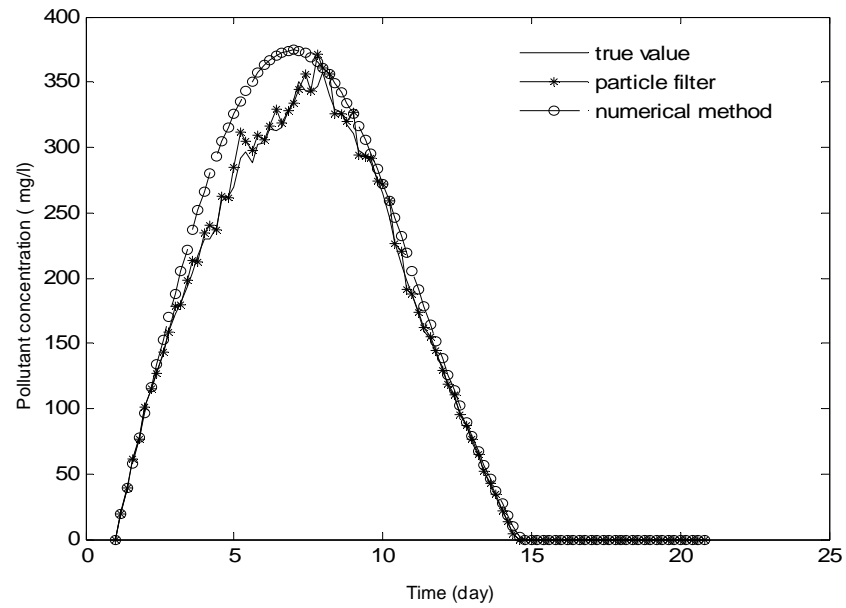

Figure 9. The concentration profile along with the time at node $=2$

\subsection{The Predicted Results at Fixed Distance}

To further investigate the involvement of concentration profile at specific locations, for example at the 2nd and 5th nodes, Figures 9 and 10 indicate the prediction varying along with the timeline at those locations. As we can see from Figures 9 and 10, the simulation result from the Particle filter matches quite well with the "true field" in each time step. The 


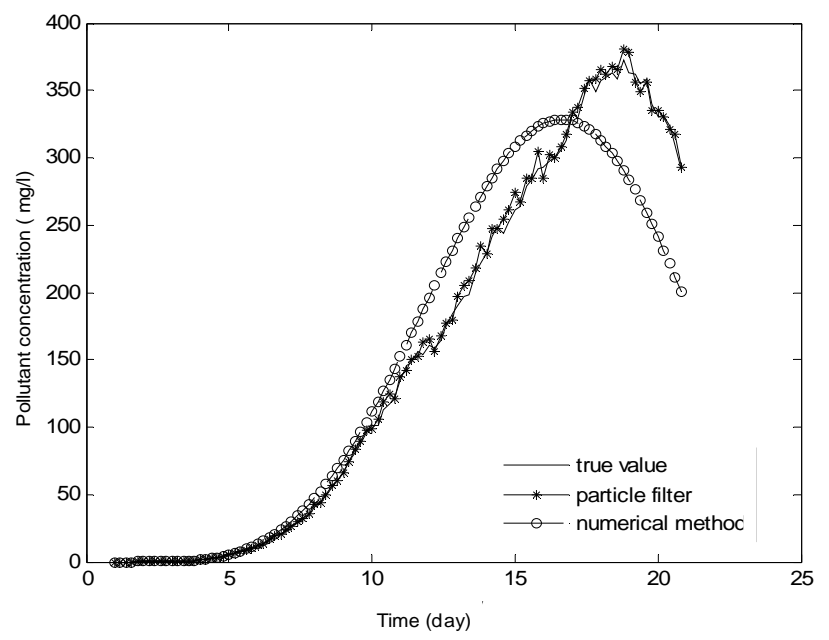

Figure 10. The concentration profile along with the time at node $=5$.

zigzag nature of the predicted concentration indicates the random error associated in the prediction process, which is reasonable but cannot be presented by the smooth curve from the numerical method.

\section{Conclusions}

The complex dynamic behavior of contaminant transport within soil environment systems has been studied using numerical methods. However, errors from the numerical method can bring unavoidable prediction deviations from the real world. Errors associated with the numerical method may include numerical errors from model mechanisms, time and space limits of numerical schemes, and boundary conditions. And the deterministic models have inaccurate transport parameters due to the complexity of the subsurface environment. According to Bayesian sequential estimate theory for the discrete-data filter problem, using the Particle filter to combine observed information into model dynamics should give more accurate estimation result compared to that from the numerical method.

This paper introduced Particle filtering for the chlorobenzene concentration estimation in a soil environment. The result shows that Particle filters are appropriate for handling the state space models describing contaminant transport. A Particle filter algorithm is demonstrated based on an advection-adsorption subsurface transport model. The method is applied to assimilate observation data and then enhances the estimation accuracy of the concentration prediction problem. The effectiveness of proposed method is then evaluated by RMSE, and promising results are obtained. It shows that with the Particle filter data assimilation, the error of the prediction is reduced by seven times or so (compared to the result from the numerical method). An extension on application of Particle filter to parameter estimation will be the subject of future work.

\section{References}

Arulampalam, M., Maskell, S., Gordon, N. and Clapp, T. (2002). A tutorial on particle filters for on-line non-linear/non-gaussian Bayesian tracking, IEEE Transactions on Signal Processing, 50, 174-188.

Beck, M.B. and Young, P.C. (1976). Systematic identification of DO-BOD model structure, J. Env. Eng. Div., 102, 909-924.

Bowles, D.S. and Greney, W.J. (1978). Steady state river quality modeling by sequential extended Kalman filters, Water Resour. Res.,12, 3281-3291.

Chang, S.Y. and Jin, A. (2005). Kalman filtering with regionalnoise to improve accuracy of contaminant transport models, J. Environ. Eng., 131, 971-982, doi:10.1061/(ASCE)0733-9372(2005)131:6 (971).

Chen, T., Julian, M. and Elaine, M. (2005). Particle filters for state and parameter estimation in batch processes, Journal of process control, 15, 665-673, doi:10.1016/j.jprocont.2005.01.001.

Cheng, X. (2000). Kalman filter scheme for three-dimensional subsurface transport simulation with a continuous input, MS Thesis, Civil Engineering, North Carolina A\&T State University, Greensboro, NC, USA.

Ching, J., Beck, J.L. and Porter, K.A. (2006). Bayesian state and parameter estimation of uncertain dynamical systems, Probabilistic Engineering Mechanics, 21, 81-96, doi:10.1016/j.probengmech. 2005.08.003.

Cosby, B.J., Hornberger, G.M. and Kelly M.G. (1984). Identification of photosynthesis-light models for aquatic systems II: Application to a macrophyte dominated stream, Ecological Modelling, 23, 2551, doi:10.1016/0304-3800(84)90117-0.

Doucet, A., de Freitas, J.F.G. and Gordon, N. (2000). Introduction to sequential monte carlo methods, Sequential monte carlo methods in practice, Springer, Berlin, 7-15.

Fernandes, L.,Warith, M.A. and Forge F.La. (1996). Modelling of contaminant transport within a marshland Environment, Waste Manage., 16, 649-661, doi:10.1016/S0956-053X(97)00006-8.

Ferraresi, M. and Marinelli, A. (1996). An extended formulation of the integrated finite difference method for groundwater flow and transport, J. of Hydrol., 175, 453-471, doi:10.1016/S0022-1694 (9 6)80020-5.

Ghil, M., Cohn, S.E.,Tavantzis, J., Bube, K. and Isaacson, E. (1981). Applications of estimation theory to numerical weather prediction, Dynamic meteorology: Data assimilation methods, Bengtsson, L., Ghil, M. and Kallen, E. eds., Springer, New York, 139-224.

Gordon, N.J., Salmond, D.J. and Smith, A.F.M. (1993). Novel approach to nonlinear/non-Gaussian Bayesian state estimation, IEE proc., 140(2), 107-113.

Gustafsson, N. (1981). A review of methods for objective analysis, Dynamic Meteorology: Data Assimilation Methods, Applied Mathematical Sciences, L. Bengtsson, M. Ghil and E. Kallen, Eds., Springer Verlag, New York, 17-76.

Harrouni, K.E., Ouazar, D., Wrobel, L.C. and Cheng, A.H.D. (1997). Aquifer parameter estimation by extended kalman filtering and boundary elements, Engineering Analysis with Boundary Elements, 19(3), 231-237, doi:10.1016/S0955-7997(97)00008-8.

Houtekamer, P.L. and Michell, H.L. (1998). Data assimilation using an ensemble Kalman filter technique, Mon. Weather. Rev., 126, 796-811.

Kalman, R.E. and Bucy, R.S. (1961). New results in linear filtering and prediction theory, J. Basic Eng., 83, 95-108.

Kitagawa, G. (1996). Monte carlo filter and smoother for non- Gaussian nonlinear state space models, J. Comput. Graph. Stat., 5, 125.

McLaughlin, D. (2002). An integrated approach to hydrologic data assimilation: interpolation, smoothing and forecasting, Adv. Water Resour., 25, 1275-1286, doi:10.1016/S0309-1708(02)00055-6.

Porter, D., Bruce, G., Jones, W., Huyakorn, P., Hamm, L. and Flach, G. 
(2000). Data fusion modeling for groundwater systems, J. Contam. Hydrol., 43, 303-335, doi:10.1016/S0169-7722(99)00081-9.

Svetlana, N.L., Kivman, G.A., Schroter, J. and Wenzel, M. (2003). Sequential weak constraint parameter estimation in an ecosystem model, J. Mar. Syst., 43, 31-49, doi:10.1016/j.jmarsys.2003.06.001.

Van Geer, F.C. (1982). An equation based theoretical approach to network design for groundwater levels using Kalman filters, Int. Assoc. Hydrol. Sci., 136, 241-250.

Whitehead, P.G. and Hornberger, G.M. (1984). Modelling Algal behavior in the river thames, Water Res., 18(8), 945-953, doi:10.10 16/0043-1354(84)90244-6.

Yu, Y.S., Heidari, M. and Guang-Te, W. (1989). Optimal estimation of contaminant transport in ground water, Water Resources Bulletin, 25, 295-300.

Zhou, Y.X., Te Stroet, C.B.M. and Van Geer, F.C. (1991) .Using Kalman filtering to improve and quantify the uncertainty of numerical groundwater simulations: 2. application to monitoring network design, Water Rsour. Res., 17(8), 1995-2006. 